\title{
Analyse inverse et modélisation d'essais pressiométriques réalisés sur un sol de Beyrouth
}

\section{A. FAWAZ}

H. EL GHOCHE

Université libanaise,

IUT de Saida, Liban

afawaz@ul.edu.Ib

h.elghoche@iutsaida.edu.Ib

Y. KAZAN

Université libanaise Faculté de génie, Roumieh Liban

ykazan@ul.edu.lb

Y. MALECOT

M. BOULON

E. FLAVIGNY

Laboratoire Sols, Solides

Structures, Grenoble

France

Yann.Malecot@hmg.inpg.fr,

Marc.Boulon@hmg.inpg.fr Etienne.Flavigny@hmg.inpg.fr

Cet article présente une série d'essais pressiométriques et pénétrométriques (dynamique SPT) sur sol sableux (quartier « Unesco » de Beyrouth). Les résultats en sont comparés en étudiant la variation des paramètres avec la profondeur. Des échantillons de ce sol ont été soumis à des essais de laboratoire. Ces données sont exploitées par modélisation numérique de I'essai pressiométrique (logiciel PLAXIS), en analyse inverse, en vue du calcul d'ouvrages géotechniques.

Mots-clés: analyse inverse, pressiomètre, pénétromètre, sable, cisaillement, fonction objectif, module, frottement interne.

\section{Inverse analysis and numerical modelisation of pressuremeter test performed in Beirut}

A series of pressuremeter and penetrometer (dynamic SPT) tests on a sandy soil of Unesco area in Beirut are presented. These results are compared by observing the variations of their parameters versus depth. Samples of this soil have been tested in laboratory. All these data are used for a numerical modelling of the pressuremeter test (software PLAXIS), by inverse analysis, for calculation of geotechnical constructions.

Key words: inverse analysis, pressuremeter, penetrometer. sand, shear, objectif function, modulus, internal friction. 


\section{Introduction}

L'essai pressiométrique est l'un des essais in situ très utilisés dans le monde, dont le succès, depuis le travail original de Ménard (1957) tient vraisemblablement à deux faits: il permet d'une part de mesurer simultanément rigidité et résistance des sols et il est associé d'autre part à une méthode de calcul des fondations (D60), Le géotechnicien dispose ainsi d'un outil de caractérisation in situ et d'un autre de dimensionnement. Parallèlement à ces travaux, des méthodes d'interprétation de l'essai pressiométrique ont été développées, en élasticité linéaire, puis en élastoplasticité monodimensionnelle ou bidimensionnelle (Salençon, 1966; Combarieu, 1995). Ces calculs visaient à exprimer la pression limite pressiométrique à partir des caractéristiques du sol. Le développement récent des méthodes numériques et la nécessité d'alimenter les logiciels de calcul d'ouvrages (en éléments finis) ont conduit à rechercher d'autres moyens d'(extraire» des essais pressiométriques les caractéristiques mécaniques des sols testés. On peut citer à cet égard Cambou et Bahar (1995) dont le logiciel Press'Ident permet d'identifier les paramètres de modèles (Duncan, Chang, 1970 et CJS).

Le présent travail s'inscrit dans une étude générale dont l'objectif est un outil d'analyse inverse pour la géotechnique, pour identifier les paramètres mécaniques de sols, à partir d'essais in situ. On présentera les résultats d'essais pressiométriques et pénétrométriques dynamiques SPT réalisés sur un sol libanais. L'identité de site, de forage, permet la comparaison des résultats des deux types d'essais. Des échantillons de sol ont de plus été prélevés en forages et soumis à des essais de laboratoire, pour comparaison. De là, l'étude numérique de lessai pressiométrique a été conduite par analyse inverse, en vue de déterminer les caractéristiques de sol à prendre en compte en calcul d'ouvrage.

\section{Étude expérimentale}

\section{1}

\section{Essais in situ}

Une série d'essais pressiométriques et SPT a été menée dans un terrain sableux du quartier « Unesco » à Beyrouth, faisant partie d'un site sédimentaire, des formations quaternaires au Liban (Kazan, 2000). Huit forages avaient été réalisés, à des profondeurs allant jusqu'à 20 à $25 \mathrm{~m}$. Dans ces forages, l'essai SPT a été exécuté tous les 1 à 2,5 m, par chute libre d'une masse de $64 \mathrm{~kg}$ d'une hauteur de chute de 0,76 m. Les essais pressiométriques ont été réalisés dans deux des forages, tous les $1 \mathrm{~m}, 1,5 \mathrm{~m}$ ou $2 \mathrm{~m}$. A partir de ces forages et des échantillons prélevés, on a pu identifier les couches de sol depuis la surface : $5 / 6 \mathrm{~m}$ de sable moyen moyennement dense, $10 / 12 \mathrm{~m}$ de sable silteux dense à très dense, $5 / 7 \mathrm{~m}$ de sable très dense ou faiblement cimenté (Fig. 1).

\section{Qsilit}

\section{Résultats des essais SPT}

Les résultats des essais SPT sont présentés sur la figure 1. Ils montrent l'augmentation du nombre de coups avec la profondeur, ce qui traduit, suivant les règles de corrélation connues, l'augmentation de la densité avec la profondeur. On note parfois la présence de poches de faible densité dans le massif, même dans les couches très denses. Les résultats du forage $n^{\circ} 1$ montrent une augmentation constante du nombre de coups $\mathrm{N}$ avec la profondeur. Les résultats des autres forages ne présentent pas toujours ce même caractère, surtout jusqu'à une profondeur de l'ordre de $10 \mathrm{~m}$ environ.

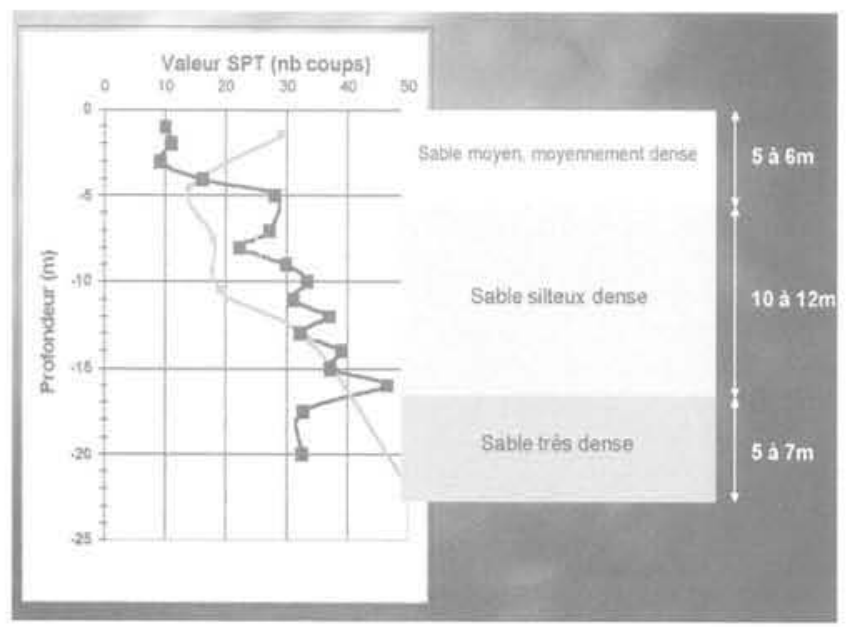

FIG, 1 Stratigraphie du site et nombre $\mathrm{N}$ de coups au pénétromètre SPT en fonction de la profondeur (圆 Forage $\mathrm{n}^{\circ} 1$; 典 Forage $\mathrm{n}^{\circ} 5$ ). Soil layers of site and variation with depth of number $\mathrm{N}$ of blows obtained from penetrometer SPT.

En utilisant les tables de corrélation existantes, on trouve un angle de frottement interne $\varphi$ variant entre $32^{\prime}$ et $40^{\circ}$ à la profondeur de $1 \mathrm{~m}$ à $7 \mathrm{~m}$; ces valeurs sont de l'ordre de grandeur de celles trouvées au laboratoire, Mais, d'une part, la détermination de $\varphi$ à partir du SPT nécessite des essais d'identification en laboratoire pour permettre l'entrée dans les tables de corrélation. D'autre part, cette détermination, non liée à une formulation théorique basée sur le comportement du sol, reste incomplète si elle n'est pas accompagnée d'autres caractérístiques mécaniques importantes telles que le module d'élasticité et l'angle de dilatance $\psi$. Le recours à l'essai pressiométrique est donc indispensable, pour déterminer toutes les caractéristiques mécaniques du sol avec une référence théorique.

\subsection{5.}

\section{Résultats des essais pressiométriques}

La croissance des caractéristiques mécaniques avec la profondeur parait plus clairement à travers les résultats des essais pressiométriques. La figure 2 présente les courbes pressiométriques obtenues à partir des essais réalisés dans le forage $n^{\circ} 2$. On peut remarquer la diminution de volume injecté, pour une même pression appliquée, ainsi que l'augmentation de pression limite, avec la profondeur. La figure 3 présente l'évolution de la pression limite avec la profondeur, on peut remarquer que cette pression varie de manière presque parabolique 
en fonction de la profondeur. Cette variation parabolique a été observée au laboratoire 35 de Grenoble dans les expérimentations de Mokrani (1991) et Renoud-Lias (1978) sur le sable d’Hostun. Fawaz (1993) et ces deux auteurs ont trouvé une variation presque linéaire de la pression limite en fonction de la profondeur quand cette dernière reste faible, ce qui reflète le début de la courbe parabolique aux faibles valeurs de la profondeur.

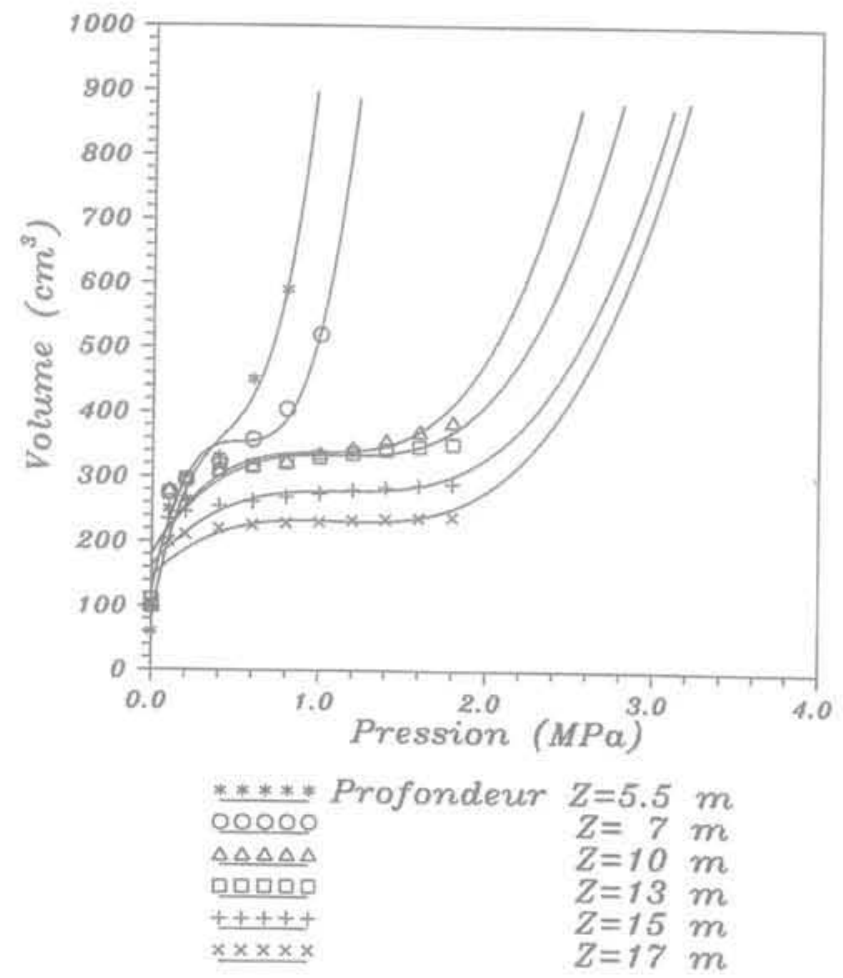

FIG. 2 Courbes pressiométriques à diverses profondeurs (forage $\mathrm{n}^{\circ} 2$ ).

Pressuremeter curves at several depths. (Borehole $n^{\circ} 2$ ).

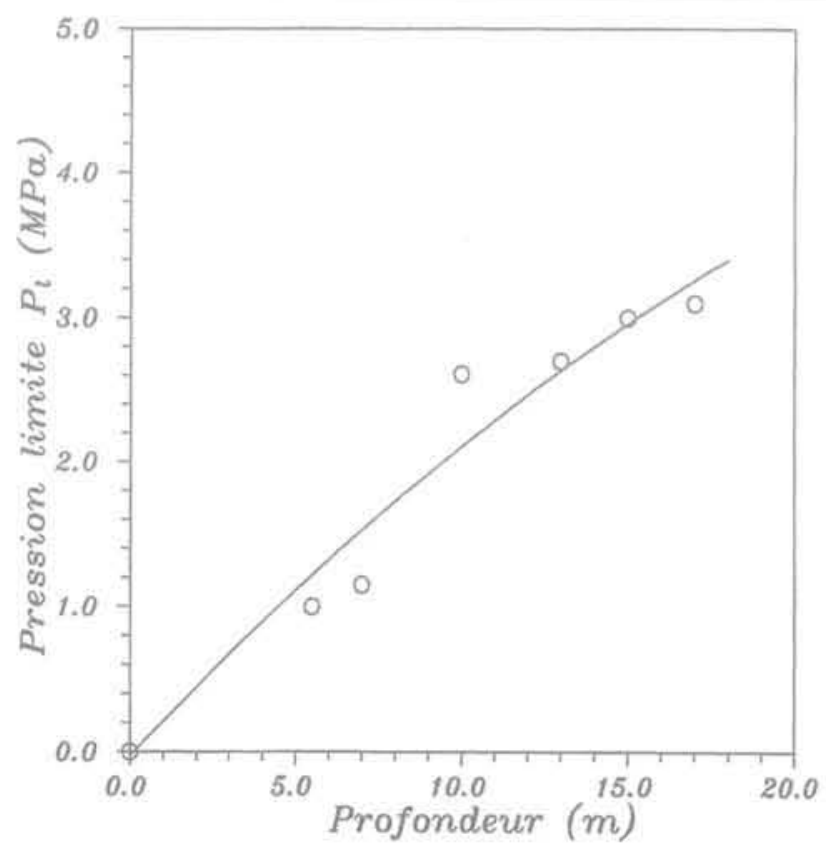

FG. 3 Variation de la pression limite avec la profondeur (forage $\mathrm{n}^{\circ} 2$ ).

Variation of limit pressure with depth (Borehole $n^{\circ}$ 2).
En traçant les courbes pressiométriques (Fig. 4) et la variation de la pression limite en fonction de la profondeur (Fig. 5) obtenues à partir du forage $n^{\circ} 5$. On retrouve le résultat obtenu avec le SPT sur la présence des poches de faible densité à l'intérieur du massif. On peut remarquer l'augmentation parabolique de la pression limite avec la profondeur, mais coupée par la présence de la poche de faible résistance.

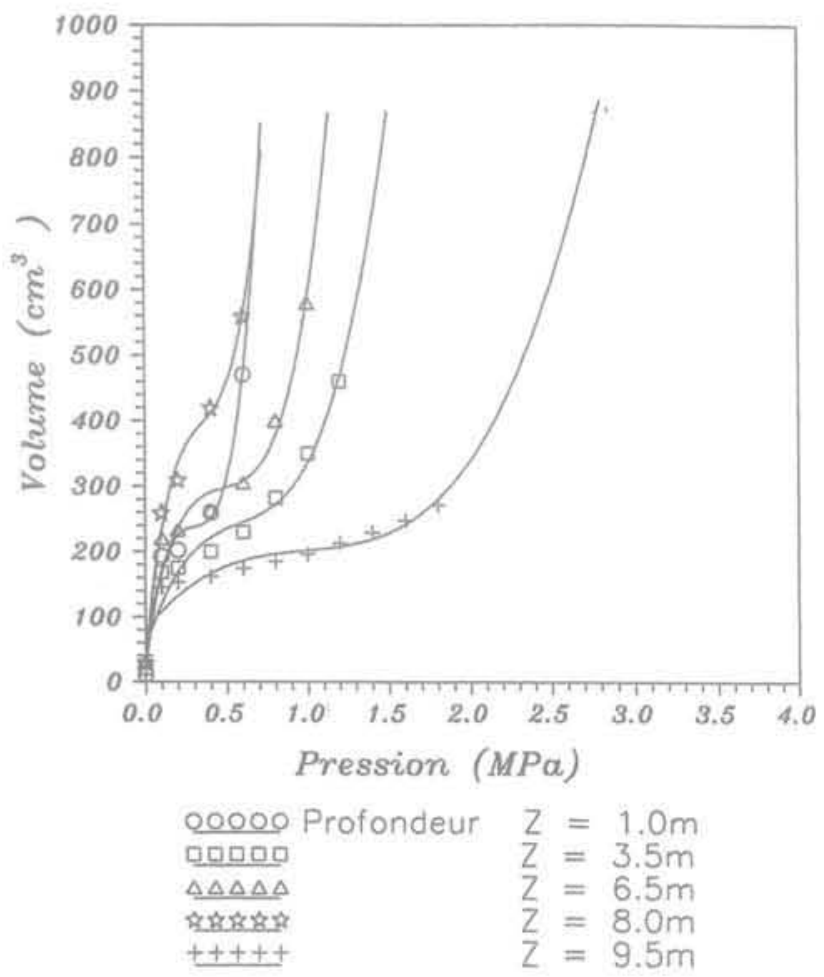

FIG. 4 Forage $n^{\circ} 5$ : courbes pressiométriques à diverses profondeurs.

Borehole $n^{\circ}$ 5: Pressuremeter curves at several depths.

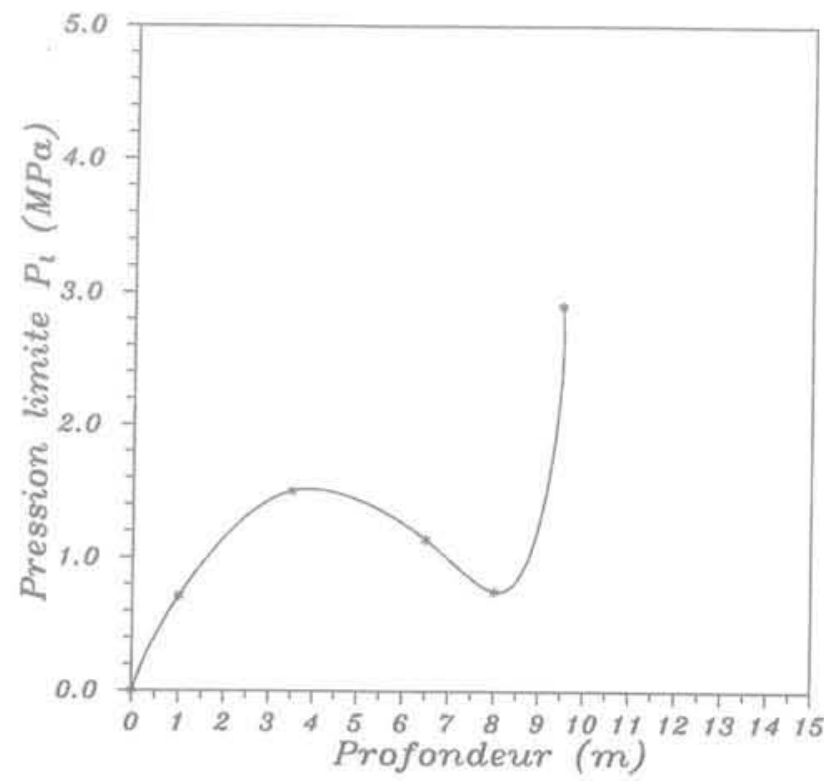

FG.5 Variation de la pression limite avec la profondeur (forage $\mathrm{n}^{\circ} 5$ ).

Variation of limit pressure with depth (Borehole $\left.n^{\circ} 5\right)$. 
Sur la figure 6 est portée l'évolution du module pressiométrique avec la profondeur, qui augmente presque linéairement avec la profondeur.

La détermination précise de la pression des terres au repos $\mathrm{P}_{0}$ demeure un exercice assez délicat. Cette pression est réputée correspondre au point d'inflexion de la courbe pressiométrique, ce qui est souvent subjectif. La figure 7 présente l'évolution de $\mathrm{P}_{\mathrm{f}}$ avec la profondeur ainsi déterminée, qui se révèle quasi-linéaire, d'où un coefficient $\mathrm{K}_{0}$ de l'ordre de 0,42 , situé dans la fourchette des valeurs de sols sableux (Fawaz, 1993; Mokrani, 1991).

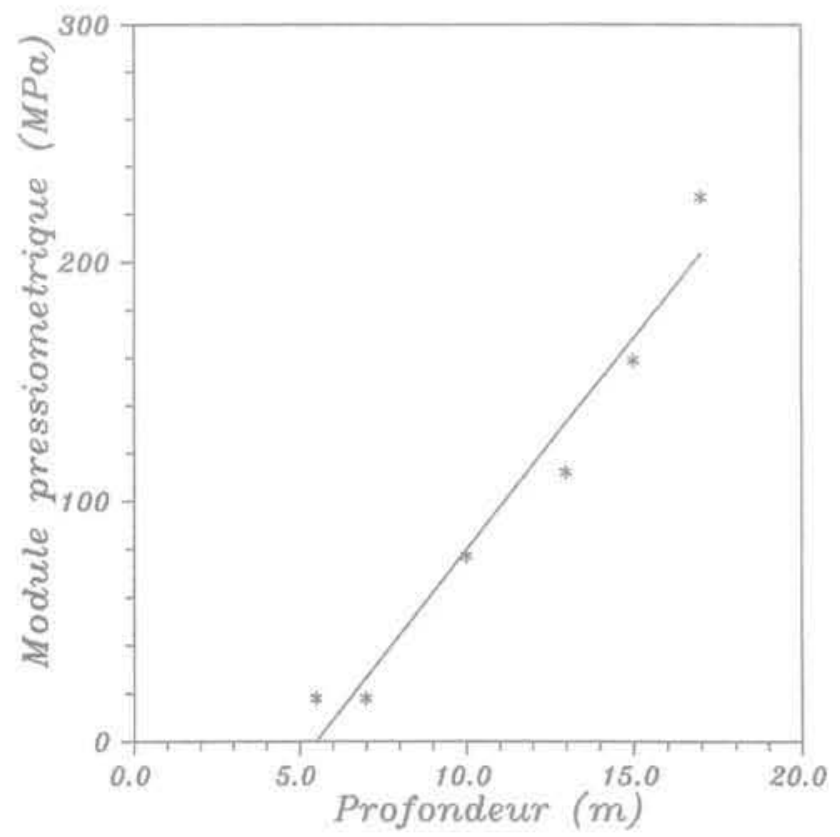

FIG.6 Module pressiométrique en fonction de la profondeur.

Evolution of pressuremeter modulus with depth.

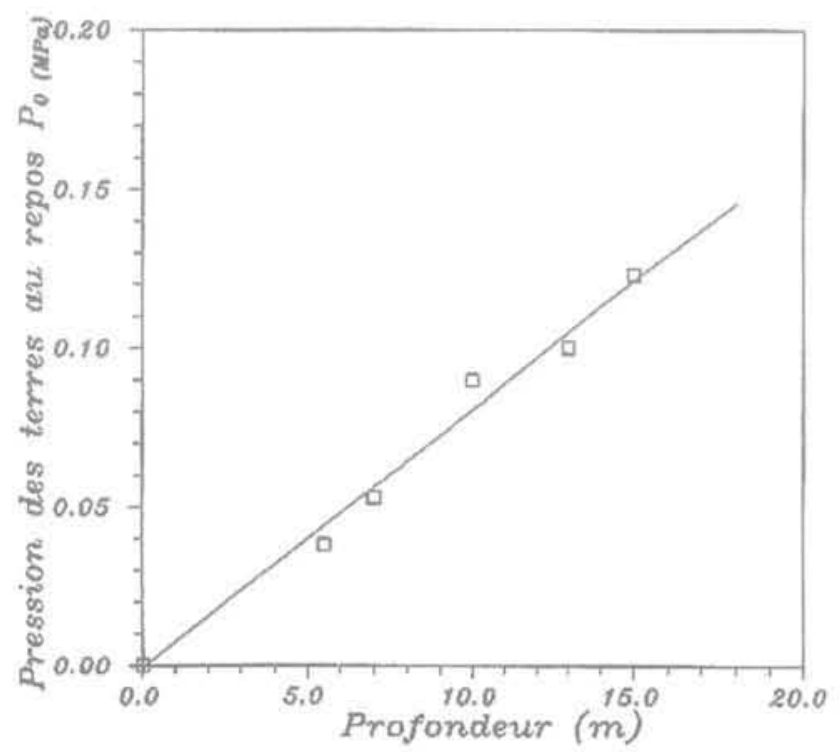

FIG. 7 Pression des terres au repos en fonction de la profondeur.

At rest earth pressure in function of depth.

\section{Essais mécaniques au laboratoire}

Les échantillons du sol ont été testés au laboratoire de l'Institut universitaire de technologie à Saida, Liban. Les résultats des essais d'identification (analyse granulométrique, limites d'Atterberg, teneur en eau) situent le matériau comme un sable moyen, avec une étendue de granulométrie comprise entre 0,13 et $11,5 \mathrm{~mm}$, un $\mathrm{d}_{50}$ de $0,25 \mathrm{~mm}$, et une teneur en eau variant entre 3 et $10 \%$. Quelques échantillons ont été soumis à un essai de cisaillement direct; la boîte est parallélépipédique de $6 \mathrm{~cm} \times 6 \mathrm{~cm} \times 2 \mathrm{~cm}$ (épaisseur), et la vitesse de cisaillement choisie a été de $5 \mathrm{~mm} / \mathrm{min}$. Ces essais ont fourni une valeur de cohésion négligeable (8 à $13 \mathrm{kPa}$ ) et un angle de frottement interne situé entre $32^{\circ}$ et $34^{\circ}$ (Fig, 8).

Droite de Coulomb

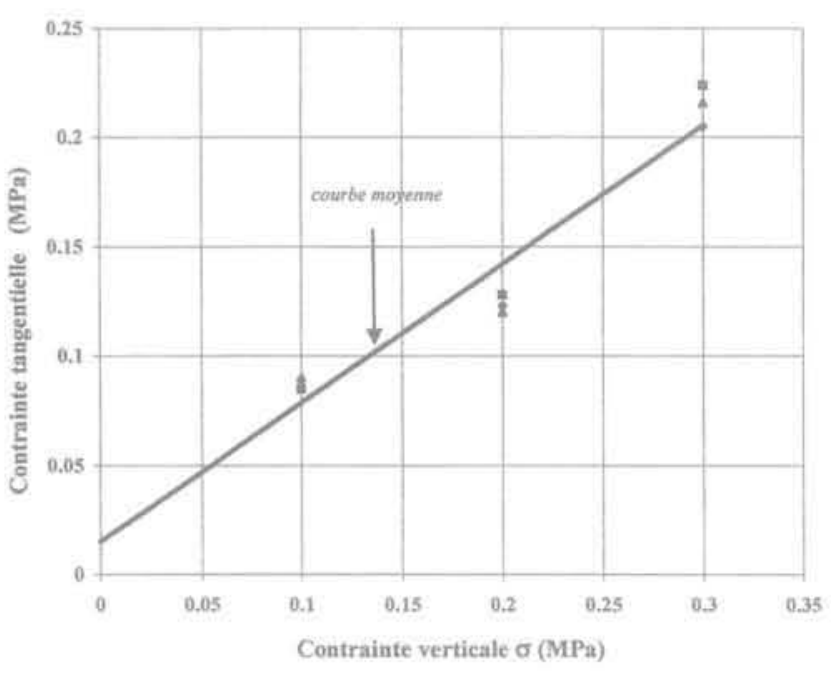

FiG. 8 Résultats des essais de cisaillement direct. Results of shear box test.

\section{Analyse inverse et identification}

L'interprétation de l'essai pressiométrique décrite précédemment permet le calcul de fondations superficielles et profondes ainsi que le calcul d'ouvrages de soutènement par des méthodes semi-empiriques qui ont été validées. En revanche celle-ci est insuffisante pour identifier les paramètres mécaniques du sol nécessaires à la réalisation d'un calcul par éléments finis. Or, dans le cas d'ouvrages complexes ou d'interactions entre ouvrages une telle modélisation est souvent indispensable. Les bureaux d'études géotechniques sont alors confrontés à leur méconnaissance des propriétés mécaniques du sol. Ce constat et le coût des essais de laboratoire conduisent à poser la problématique de l'analyse inverse en géotechnique: quelle information concernant les paramètres de sols est-il possible de déduire de résultats de mesures in situ?

Pour répondre à cette question, il faut être capable de résoudre un problème inverse non linéaire dont les conditions aux limites sont en général complexes. Bien que la présente étude soit limitée à l'interprétation de 
l'essai pressiométrique, afin d'avoir une méthode d'identification capable de s'adapter à d'autres types de mesures in situ, essais ou ouvrages instrumentés, nous avons choisi de résoudre ce problème inverse en utilisant une approche directe (Gioda et Maier, 1980; Gens et al., 1988; Lecampion et al., 2002). Cette méthode d'identification est brièvement présentée dans le paragraphe suivant. Elle est ensuite appliquée à un résultat d'essai pressiométrique réalisé sur le sol de Beyrouth.

\section{1}

\section{Méthode d'identification}

La méthode utilisée a été proposée par Malécot et al. (2003). Elle consiste à choisir un jeu de paramètres de sol a priori. Ce jeu de paramètres, noté vecteur paramètre p à $N_{p}$ composantes, est utilisé comme donnée d'entrée pour résoudre numériquement le problème direct associé à l'aide du logiciel PLAXIS. Il est ensuite ajusté de manière à ce que les mesures in situ soient correctement reproduites par la simulation numérique. D'un point de vue pratique, il s'agit, dans un premier temps, de choisir une mesure objective de l'écart entre les mesures et la simulation, puis d'établir un algorithme judicieux de minimisation de cet écart.

L'écart entre les $N$ points de mesure expérimentaux Ue et les résultats numériques associés Un est évaluée quantitativement par une fonction erreur scalaire $F_{\text {err }}$ au sens de la méthode des moindres carrés. La fonction $F_{\text {sf }}(\mathrm{p})$ étant définie pour tout vecteur $\mathrm{p}$ donné, le problème inverse est ensuite résolu comme un problème de minimisation dans l'espace des paramètres ( $N$ dimensions). Afin d'avoir une méthode capable de s'adapter à des fonctions erreurs non convexes, l'algorithme utilisé est basé sur une méthode de gradient. Etant donné un jeu de paramètres a priori $\mathrm{p}$, la fonction erreur $F_{\text {ef }}\left(\mathrm{p}^{\prime}\right)$ et son gradient $\nabla F_{\text {( }}$ ( $)$ sont évalués par simulations successives du probleme direct associé. Le point de calcul suivant $p^{i+1}$ est ensuite choisi tel que, $p^{i+1}=p^{i}+x d^{i}$, où d est le vecteur qui indique la direction de plus grande pente, et où $x$ est un scalaire adimensionnel qui donne le pas de descente. La valeur optimale du pas de descente est déterminée par une évaluation quadratique de $F_{\text {err }}$ dans la direction d.

\section{2}

\section{Application à un résultat d'essai pressiométrique réalisé sur le sol de Beyrouth}

Afin d'appliquer l'algorithme présenté au paragraphe 3.1 nous avons simulé l'essai pressiométrique du forage $n^{\circ} 5$ réalisé à $3,5 \mathrm{~m}$ de profondeur. La figure 9 montre la géométrie, les conditions aux limites et le maillage du modèle. Afin de réduire au minimum le nombre de paramètres de sols à identifier, nous avons utilisé un modèle de Mohr-Coulomb en supposant connus la cohésion $(c=15 \mathrm{kPa})$, le coefficient de Poisson $(v=0,3)$ ainsi que les relations empiriques suivantes : angle de dilatance $\psi=\varphi-30^{\circ}$; coefficient des terres au repos $K_{0}=1-\sin \varphi$. Le module élastique $E$ et l'angle de frottement restent alors les seuls paramètres à identifier.

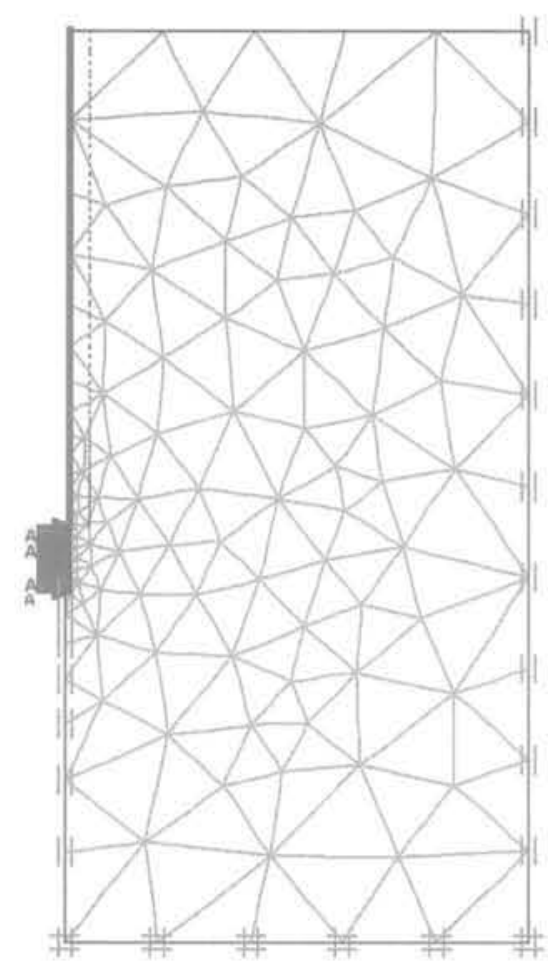

FiG. 9 Géométrie du modèle axisymétrique. Geometry of axisymetric model.

La courbe pressiométrique mesurée in situ est présentée sur la figure 10. La fonction erreur $F_{\text {cor }}(E, \varphi)$ ainsi que le chemin suivi lors du processus de minimisation sont représentés sur la figure 11. On constate que la surface de $F_{\text {ur }}$ est traversée par une vallée parfaitement plate. Elle ne possède pas un minimum unique. Le point de convergence de l'algorithme de minimisation, atteint très rapidement, dépend ainsi étroitement de la position du premier point de calcul. La figure 10 montre les courbes pressiométriques obtenues à partir de deux jeux de paramètres très distincts et minimisant $F$. On vérifie à la fois que les courbes simulées sont peu dissociables et qu'elles reproduisent bien la courbe expérimentale.

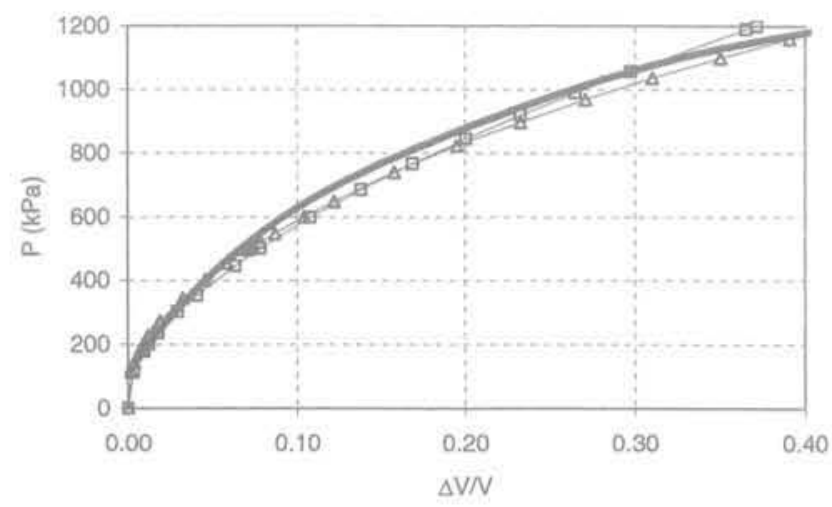

FIG. 10 Courbe pressiométrique (trait gras) mesure in situ;

(घ) $\mathrm{E}=108700 \mathrm{kPa}, \varphi=34,1^{\circ} ;(\Delta) \mathrm{E}=$ $171200 \mathrm{kPa}, \varphi=29,3^{\circ}$.

Pressuremeter curve: (bold line) in situ measurement:

(D) $\mathrm{E}=108,700 \mathrm{kPa}, \varphi=34,1^{\circ} ;(\Delta) \mathrm{E}=$ $171,200 \mathrm{kPa}, \varphi=29,3^{\circ}$, 

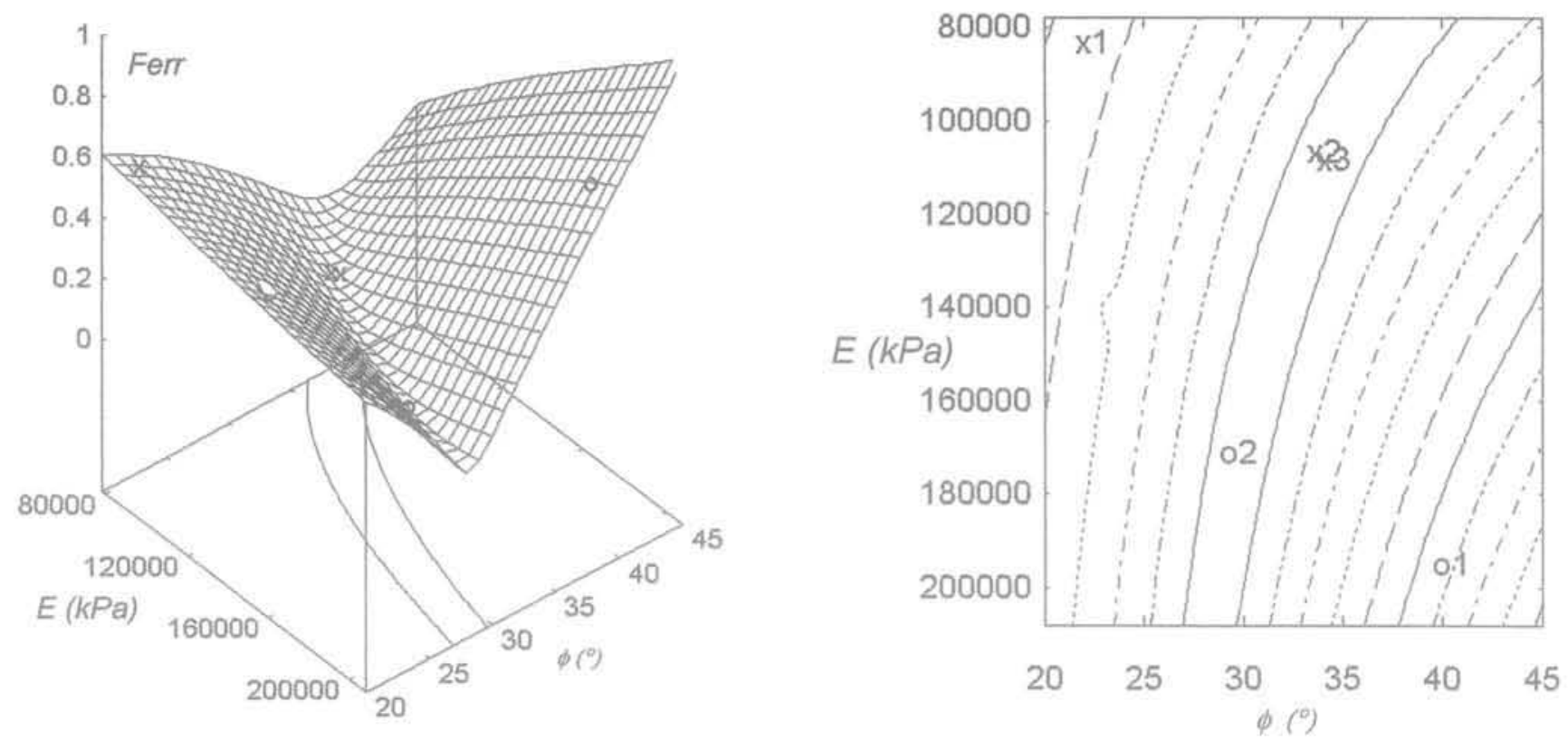

FG. $11 \quad F_{\text {err }}(E, \varphi)$ et chemins suivi par l'algorithme de minimisation;

(o1) point initial $\mathrm{E}=195000 \mathrm{kPa}, \varphi=40^{\circ} ;(x 1)$ point initial $\mathrm{E}=83200 \mathrm{kPa}, \varphi=22^{\circ}$.

$F_{\text {. }}(\mathrm{E}, \varphi)$ and paths described by the algorithm of minimisation;

(o1) initial point $E=195,000 \mathrm{kPa}, \varphi=40^{\circ} ;(x 1)$ initial point $E=83,200 \mathrm{kPa}, \varphi=22^{\circ}$.

\section{4}

\section{Conclusion}

Les informations pouvant être directement tirées de l'essai pressiométrique sont considérables (pression des terres au repos, pression limite conventionnelle, module pressiométrique). Pourtant ces paramètres ne suffisent pas comme données d'entrée pour réaliser un calcul d'ouvrage par éléments finis. Afin de compléter l'interprétation de cet essai nous avons tenté d'en faire une analyse inverse par simulation numérique directe. L'algorithme adopté fonctionne et le modèle de MohrCoulomb permet de bien reproduire la courbe pressiométrique mesurée in situ. Cependant, il ne semble pas possible d'identifier plus d'un paramètre de sol à partir de cette courbe. Il faut choisir entre l'identification du module d'Young $E$ ou de celle de l'angle de frottement $\varphi$.

\section{Bibliographie}

Cambou B., Bahar R. - Utilisation de l'essai pressiométrique pour l'identification de paramètres intrinsèques du comportement d'un sol. Revue française de géotechnique, $n^{\circ}$ 63, 1993, p. $39-50$,

Combarieu O. - L'essai pressiométrique et la résistance au cisaillement des sols. Bulletin de Liaison des LPC 196, marsavril 1995, p. 43-50.

Duncan J.M. Chang C.H - Non linear analysis of stress and strain in soils. J. of the Soils Mechanics and Foundation Division, Proc. of the ASCE, vol. 96, $\mathrm{n}^{\circ}$ SM5, sept. 1970.

Fawaz A. - Etude expérimentale et numérique du pressiomètre en milieu pulvérulent. Thèse de Doctorat, INPG, Grenoble, 1993.

Gens A., Ledesma A., Alonso E. - Back analysis using prior information Application to the staged excavation of a cavern in rock. In Swoboda (ed.).
Numerical Methods in Geomechanics. Innsbruck Balkema, Rotterdam, 1988

Gioda G. Maier G. - Direct search solution of an inverse problem in elastoplasticity: Identification of cohesion, friction angle and in situ stress by pressure tunnel tests. International Journal for Numerical Methods in Engineering. vol. 15,1980 , p. 1823-1848.

Kazan Y. - Géologie du Liban vue par un géotechnicien. impact sur les modes de soutènement à Beyrouth. Revue française de géotechnique, $n^{5} 100,2000$, p. 75-82.

Lecampion B., Constantinescu A., Nguyen Minh D. - Parameter identification for lined tunnels in viscoplastic medium International Journal for Numerical and Analytical Methods in Geomechanics, 26, 2002, p. 1191-1211

Malécot Y., Flavigny E., Boulon M. - Identification of soil parameters for finite element simulation of geotechnical structures : pressuremeter test and excavation problem. Proc. of the 2nd Int. Conf. in structural engineering and construction, ISEC03, Rome, Balkema 2003, p. 2393-2400.

Ménard L. - Mesure in situ des propriétés physiques des sols. Annales des ponts et chaussées, vol. 14, 1957, p. 357-377.

Mokrani L - Simulation physique du comportement des pieux á grande profondeur en chambre de calibration. Thèse de Doctorat, INPG. Grenoble, 1991.

PLAXIS, Finite Element Code for Soil and Rock Analyses http://www.plaxis. nilie.html,

Renoud-Lias B. - Etude du pressiomètre en milieu pulverrulent. Thèse de Doctorat, Université Joseph-Fourier, Grenoble, 1978.

Salencon J. - Expansion quasi statique d'une cavité cylindrique ou sphérique dans un milieu élastoplastique. Annales des Ponts et chaussées, vol. 3, 1966 . p. 175-187. 